\title{
TECHNICAL OPTIONS IN RECONSTRUCTION OF THE SUPERIOR VENA CAVA ASSOCIATED WITH INVASIVE THYMIC TUMOR
}

\author{
Susumu Ishikawa, Yoshimi Otani, Ichiro Yoshida, \\ Masayuki Sugano, Osamu Kawashima, Mitsuhiro Kamiyoshihara, \\ Toru Takahashi, Yasushi Sato, Jun Mohara, \\ Yasuo Morishita \\ Second Department of Surgery, Gunma University Faculty of Medicine
}

\begin{abstract}
Six patients who underwent a reconstruction of the superior vena cava (SVC) area were reviewed. The mean age of the patients was 50 years. Only one patient complained of preoperative SVC syndrome. Pathologic diagnoses were invasive thymoma in five patients including two redo cases, and thymic carcinoid tumor in one patient. Polytetrafluoroethylene (EPTFE) grafts were used for vein reconstruction. The left innominate vein was reconstructed in all six patients. The right innominate vein and the SVC were reconstructed in three patients. Extracorporeal circulation (ECC) was used in one patient with a tumor extension into the right atrium. All patients except for one with a thymic carcinoid tumor received both postoperative irradiation therapy and chemotherapy. Two patients died during the postoperative late periods and four patients survived for three to 18 years (mean; 10) after the initial operation. Three out of four patients underwent additional surgery during the postoperative late periods. The graft patency rate was $67 \%$ in the SVC area and 50\% in the left innominate veins. In conclusion, a radical operation with reconstruction of SVC is treatment of choice for long-term survival and bilateral venous reconstruction is recommended The indication of ECC for tumor resection is still controversial.

Key words: SVC syndrome, Invasive thymoma, Venous reconstruction, Bypass grafting, Extracorporeal circulation
\end{abstract}

(Kitakanto Med.J. 51 (3) : 189 192, 2001)

\section{INTRODUCTION}

Graft replacement or patch angioplasty of the superior vena cava (SVC) should be part of planning and execution of radical excision with curative intent of advanced mediastinal tumors. Our operative results of advanced mediastinal tumor with SVC reconstruction were reviewed with particular focus on intraoperative circulatory maintenance and indication for extracorporeal circulation (ECC)

\section{MATERIALS \& METHODS}

Six patients who underwent a reconstruction of the SVC area for a ten-year period commencing in 1990 were reviewed. The mean age of the patients was 50 years old ranging from 39 to 74, including three males and three females. Only one patient complained of preoperative SVC syndrome. Pathologic diagnoses were invasive thymoma in five patients including two redo cases, and thymic carcinoid tumor in one patient. Operations were performed under median sternotomy in all cases. Tumors were resected in five patients, however, one patient with an unresectable invasive thymoma underwent bilateral bypass grafting between the bilateral innominate veins and the right atrium. SVC including bilateral innominate veins was reconstructed with expanded polytetrafluoroethylene (EPTFE) grafts using an external ring support, 8 to

Received : March 14, 2001

Address : SUSUMU ISHIKAWA

Second Department of Surgery, Gunma University Faculty of Medicine,

3-39-22 Showa-machi, Maebashi, Gunma 371-8511, Japan 
$13 \mathrm{~mm}$ in diameter. The left innominate vein was reconstructed in all six patients. Bypass grafting between the right innominate vein and the SVC was performed in three patients (Figure 1). Extracorporeal circulation (ECC) associated with electrical ventricular fibrillation was used in one patient with tumor invasion into the right atrium (Figure 2). All patients except for one with a thymic carcinoid tumor received both postoperative irradiation therapy and chemotherapy with cisplatin and doxorubicin hydro-

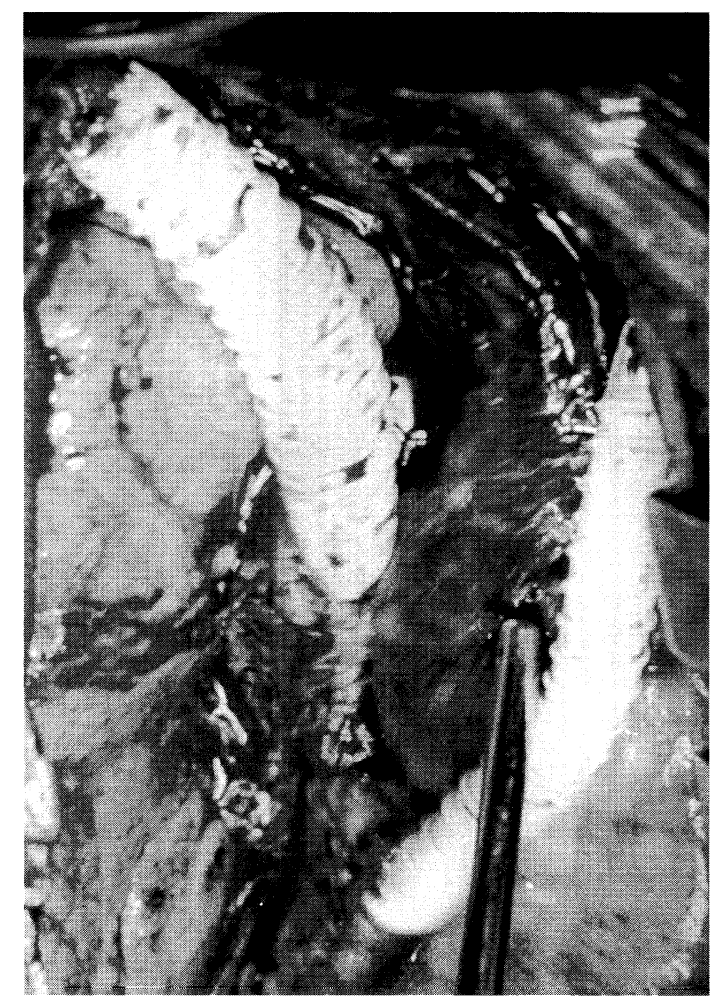

Figure 1 Bilateral venous reconstruction using expanded polytetrafluoroethylene (EPTFE) grafts with external ring supports. cloride (Adriamycin).

\section{RESULTS}

All patients survived operations without intraoperative circulatory instability or major postoperative morbidities. Two patients died of tumor advancement and recurrence, respectively, during postoperative late periods. One patient with an unresectable thymoma died two months after surgery. The other patient with a thymic carcinoid tumor died at 13 months after surgery. Four patients survived for three to 18 years (mean; 10) after the initial operation. Three out of four patients received additional surgery during postoperative late periods. Two patients underwent surgery due to pleural dissemination or tumor recurrence in the anterior mediastinum, respectively. One patient who had undergone tumor resection under ECC received additional therapy for postoperative distant metastasis within six months after the initial surgery, including local resection for a neck metastasis and irradiation therapy for a kidney metastasis (Table 1).

In the follow-up study of bypass grafts, two out of three grafts for SVC and the right innominate vein were patent. Three out of six grafts for the left innominate vein were patent. No SVC syndrome has occurred in the four long-term survivors (Table 2).

\section{DISCUSSION}

Replacement of the SVC with EPTFE grafts $^{1,2)}$ in the presence of a space-occupying lesion has been widely performed and accepted as an effective procedure $^{3)}$. The prognosis of SVC reconstruction depends upon the primary diseases, and better results have been obtained in patients with invasive thymoma than in patients with lung cancer ${ }^{4)}$. Operative outcomes of patients with SVC syndrome are generally grave.

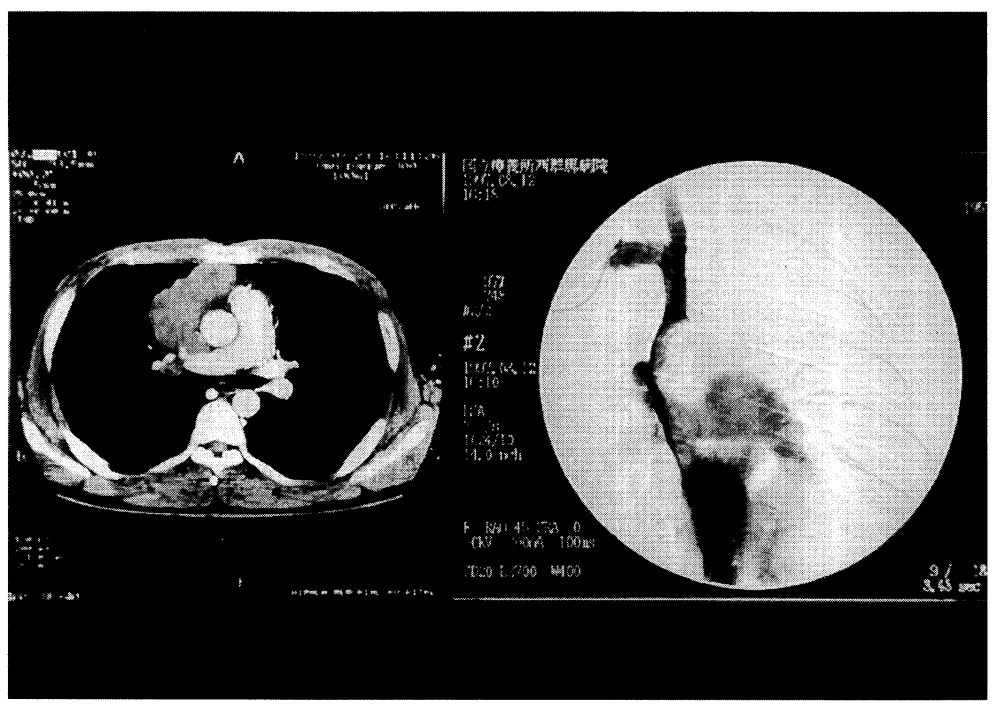

Figure 2 Preoperative $\mathrm{X}$-ray findings in patients with intracardiac invasion into the right atrium. 
Reconstruction of Superior Vena Cava

Table 1 Operative Results

\begin{tabular}{|c|c|c|c|c|c|c|}
\hline \multirow{2}{*}{ Case } & \multirow{2}{*}{ Age/ Sex } & \multirow{2}{*}{ Diagnosis } & \multirow{2}{*}{$\begin{array}{l}\text { Operation } \\
\text { (Tumor) }\end{array}$} & \multicolumn{2}{|c|}{ Postoperative } & \multirow{2}{*}{ Results } \\
\hline & & & & Chemotherapy & Irradiation & \\
\hline 1) & $55 / \mathrm{M}$ & thymoma & not-resected & - & - & Dead (2Mo) \\
\hline 2) & $74 / \mathrm{M}$ & carcinoid & resected & - & - & Dead (13Mo) \\
\hline 3) & $40 / F$ & thymoma & resected & CDDP, ADR & + & Alive (18Y) \\
\hline 4) & $47 / F$ & thymoma & resected & CDDP, ADR & + & Alive (10.5Y) \\
\hline 5) & $47 / F$ & thymoma & resected & CDDP, ADR & + & Alive (8Y) \\
\hline 6) & $39 / \mathrm{M}$ & thymoma & resected(ECC) & CDDP, ADR & + & Alive (3.5Y) \\
\hline
\end{tabular}

Abbreviations

M ; male, F ; female, ECC ; extracorporeal circulation, CDDP ; cisplatin, ADR ; Adriamycin, Mo ; month, Y ; year

Table 2 Graft Patency

\begin{tabular}{|c|c|c|c|}
\hline Position & Diameter & Pater & cy $(\%)$ \\
\hline Left & & Total & $3 / 6(50)$ \\
\hline \multirow{2}{*}{ Innoiminate vein } & $10 \mathrm{~mm}$ & & $1 / 2$ \\
\hline & $8 \mathrm{~mm}$ & & $1 / 2$ \\
\hline Right & & Total & $2 / 3(67)$ \\
\hline SVC/ & $13 \mathrm{~mm}$ & & $1 / 1$ \\
\hline Innoiminate vein & $8 \mathrm{~mm}$ & & $1 / 2$ \\
\hline
\end{tabular}

Abbreviations SVC: superior vena cava

Lochridge and his colleagues ${ }^{5)}$ reported that patients with upper airway obstruction and/or cerebral edema survived only 1.4 months. In our study, a patient with SVC syndrome died 13 months after surgery due to the recurrence of thymic carcinoid. Four patients survived for three to 18 years after the initial operation in spite of pleural dissemination or tumor recurrence. Longterm survival was obtained in patients with a lowgrade malignant thymoma with complete resection followed by adjuvant chemotherapy, irradiation and additional surgery.

SVC obstruction caused by tumors generally has a slow progression and is associated with collateral venous channels. Unilateral venous reconstruction is sometimes considered to be an adequate procedure, however, we principally select a bilateral venous reconstruction. Reconstruction of the left-side venous return by grafting between left innominate vein and right atrial appendage is an initial procedure. Larsson and his colleagues ${ }^{6)}$ reported the usefulness of an intraoperative temporary bypass. No circulatory instability occurred in our patients with bilateral venous reconstruction. Late graft patency is not always excellent in patients with bilateral venous reconstruction. Okada and his colleagues ${ }^{7)}$ reported that graft patency rate was $82 \%$ in the right side and $38 \%$ in the left. In our study, graft patency rate was $67 \%$ in the right side and $50 \%$ in the left. Bilateral venous reconstruction is the treatment of choice in order to maintain circulatory stability during surgery and to reduce the occurrence of SVC syndrome at the time of tumor recurrence.

Indication for ECC for tumor resection is still controversial. Radical excision with ECC could ensure near-total removal of the mass and prevent early death from cardiovascular complications, particularly in patients with intracardiac tumor extension into the right atrium ${ }^{8}$. Our patient who underwent tumor resection during ECC received additional therapy for two distant metastases occurred within six months after the initial surgery, which might be due to the systemic dissemination of tumor cells by ECC. In conclusion, a radical operation with reconstruction of SVC is effective for long-term survival, and bilateral venous reconstruction is recommended to maintain circulatory stability during surgery.

\section{REFERENCES}

1) Shimizu N, Moriyama $S$, Aoe $M$, et al. The surgical treatment of invasive thymoma. Resection with vascular reconstruction. J Thorac Cardiovasc Surg 1992; 103 : 414-420.

2) Nakahara $K$, Ohno $K$, Hashimoto $J$, et al Thymoma. Results with complete resection and adjuvant postoperative irradiation in 141 consecutive patients. J Thorac Cardiovase Surg 1988; 95: 1041-1047.

3) Lochridge SK, Knibbe WP, Doty DB. Obstruction of the superior vena cava. Surgery $1979 ; 85$ : 14-24.

4) Fujiwara, Y Cohn LH, Adams D, et al. Use of Gortex grafts for replacement of the superior and inferior venae cavae. J Thorac Cardiovasc Surg $1974 ; 67$ : 774-779.

5) Dartevelle $P$, Chapelier A, Navajas $M$, et al. Replacement of the superior vena cava with polytetrafluoroethylene grafts combined with resection of mediastinal-pulmonary malignant tumors. Report of thirteen cases. J Thorac Cardiovasc Surg 1987; 94 : 361-366.

6) Larsson S, Lepore V. Technical options in reconstruction of large mediastinal veins. Surgery $1992 ; 111: 311-317$.

7) Okada M, Sugimoto $T$, Yamamoto H. Surgical strategy for invasive pulmonary and mediastinal 
tumors requiring superior vena cava reconstruction (in Japanese with English abstract). Kyobu Geka 1999; 52 : 14-18.

8) Okereke OU, Nzewi OC, Chikwendu VC, Odigwe E, Onuigbo WB. Radical excision of invasive thymoma with intracardiac extension. J Cardiovasc Surg 1994; 35 : 355-358. 